
\title{
3 Research Square \\ Effects of the Liaison Nurse Management on the Infectious Stroke Complications: A Randomized Controlled Trial
}

\section{Zohreh Kalani}

Shahid Sadoughi University of Medical Sciences and Health Services

Sedigheh Ebrahimi ( $\sim$ ebrahimi.mi222@gmail.com)

Shahid Sadoughi University of Medical Sciences and Health Services

Hossein Fallahzadeh

Shahid Sadoughi University of Medical Sciences and Health Services

\section{Research Article}

Keywords: nurse, pneumonia, stroke, urinary tract infection.

Posted Date: February 26th, 2021

DOI: https://doi.org/10.21203/rs.3.rs-228901/v1

License: (1) This work is licensed under a Creative Commons Attribution 4.0 International License.

Read Full License

Version of Record: A version of this preprint was published at BMC Nursing on January 20th, 2022. See the published version at https://doi.org/10.1186/s12912-021-00802-0. 


\section{Abstract \\ Background}

Two of the most serious complications after stroke are pneumonia, and urinary tract infection. Liaison nurse, from hospital admission to discharge and then at home helps patients with complicated caring issues stroke. This study investigates the effect of liaison nurse management on the incidence of pneumonia and urinary tract infection in patients with stroke after discharge from the hospital.

\section{Methods}

This randomized controlled trial was conducted on 80 patients in a hospital in Iran. The intervention group was assessed and developed a caring program by the liaison nurse and the control group received routine care. Two weeks and two months after discharge, the patients were evaluated for the incidence of pneumonia and urinary tract infection. Collected data were analyzed using the Chi-square test. $\mathrm{P}<0.05$ was considered statistically significant.

\section{Results}

The two groups were homogenous in terms of mean age; gender frequently distribution, and having urinary catheter. The incidence of pneumonia in intervention and control groups $(11.6 \%$ vs. $19.2 \%, P=$ 0.35 ) had no statistically significant differences, but there was a significant difference in the incidence of urinary tract infection ( $0 \%$ vs. $24.6 \%, \mathrm{P}<0.001)$.

\section{Conclusions}

With liaison nurse performance, there was a significant difference in the incidence of urinary tract infection, in two months after discharge from hospital, but the incidence of pneumonia had no statistically significant differences in two groups. Nurse's evaluation each patient individually according to needs, developing and monitoring the home-based care program, beyond overall education to these patients, could reduce some of complications of a stroke.

\section{Trial registration:}

This study is retrospectively registered by Iranian Registry of Clinical Trials with decree code: IRCT20170605034330N3 on April 4, 2018.

\section{Background}


Stroke is the leading cause of functional impairments in adults which could affects both patients and their relatives in most regions. It is estimated to be a major health problem in the Middle East (1-2), and in Iran is greater than in most western countries, with stroke occurring at younger ages (3).

Stroke is defined broadly as central nervous system infarction, which is brain, spinal cord, or retinal cell death attributable to ischemia, based on pathological, imaging, other objective evidence, and/or clinical evidence (4).

Despite recent advances in emergency treatment of stroke and reduction of mortalities, it remains a major cause of mortality and disability (5). The quality of care for stroke survivors is still lacking, especially in patients who are transported directly home. Patients face significant barriers to recovery and an independent life, including cognitive and physical limitations, multiple medications, and a lack of social support (6).

Two thirds of patients experience at least one complication during the first week after stroke and 4 of 5 during three months of follow-up (7). Complications of direct or indirect stroke influence patients' therapeutic outcomes including duration of hospitalization and mortality rate (8).

Two of the most serious complications after stroke are pneumonia, which occurs in $2.4-47.4 \%$ of patients (9) and urinary tract infection (UTI), which range from 1 to $24 \%$ in the week and in the first month after stroke (10).

Regarding the prevalence of complications in patients with stroke, comprehensive nursing care is very required for reducing the severity of complications, disability, and mortality within weeks after stroke (5). Unfortunately, the gap between the hospital and patient support systems in the community leads to a decline in the quality of care and its continuation (11). Improvement in quality of care, e.g. by a better preparation of the patient for discharge, is also an important aim in liaison nursing (12).

The discharge process from the hospital and his/her transfer to the home for the patient and the hospital is very important and should be performed gradually. Continuation of nursing interventions by the special stroke nurse as a liaison nurse after discharge has been beneficial for the patient and family through focusing on education and support (13). Liaison nurse providing nursing services after discharge from the hospital, especially in chronic patients, will reduce readmission to hospital and thus continuity of care. Liaison nurse, as a new role for nurses, has a positive effect on the quality of a patient's outcome, from hospital admission to discharge and then at home (14). Some health services in the world focus on this new role of the nurses adopted a terminology, such as "nurse liaison" or "discharge management nurse" (15).

The liaison nurse in coordination with other members of the healthcare team ensure that all patients and their families are taken into consideration, nursing care and treatment are organized for each patient, and prevention of separate complications is performed (5). 
The liaison nurse service was introduced in Australian hospitals to improve post-discharge care from the intensive care unit, reduce complications and hospitalization, and hospital mortality. The liaison nurse is expected to have enough knowledge and skills to continue patient care and communicate with other nurses and improve the patient's outcomes (14). The role of the liaison nurse is to improve the patient's pre-discharge care plan, improve the relationship between hospital staff and healthcare staff in the community, and provide home care facilities with the help of hospital staff (17).

Liaison nurses through careful and comprehensive examination and the use of communication and technical skills can help with care in patients with multiple problems. (18). The findings of a systematic review in Iran stressed the role of the liaison nurse in improving the outcomes of patient care after discharge from the intensive care unit, and suggested further research to provide more evidence on the concept of the liaison nurse (19). In the only study found on liaison nursing for stroke patients, the researchers suggested the liaison nurses have been moderately successful in their jobs and therefore recommended future research on liaison nursing (12).

This study is one of the first studies about liaison nursing in Iran and aimed to investigate the effect of liaison nurse performance on the incidence of complications in patients with stroke after discharge from the hospital.

\section{Methods}

\section{Study design and settings}

This randomized clinical trial was performed in parallel in patients with stroke in March 2018 to February 2019. This study was done in neurology department of Ali-Asghar hospital in Shiraz, an urban area of Iran and after discharge, at patients' home.

\section{Study population and sample}

Patients were selected through convenience sampling. The inclusion criteria included age 45-85 years and a score of 16-25 based on the National Institutes of Health Stroke Scale (NIHSS), which strongly recommend for assessment of the stroke recovery (20). The Cronbach's alpha coefficients obtained for determining the internal consistency reliability of the Persian version of NIHSS were 0.893 for the entire group of patients (21). Exclusion criteria were the presence of pneumonia, UTI and immobility before the onset of stroke.

Based on reported incidence rate for UTI (7); $\mathrm{a}=0.05, \beta=0.2$; with a view to reducing the incidence of UTI by $20 \%\left(p_{1}=21 \%\right.$ and $\left.p_{2}=1 \%\right)$; we used the following formula to calculate the sample size for comparing ratios in two independent groups. $n=\left(Z_{a / 2}+Z_{\beta}\right)^{2} \times\left[p_{1}\left(1-p_{1}\right)+p_{2}\left(1-p_{2}\right)\right] /\left(p_{1}-p_{2}\right)^{2}$

Considering the high limitations for referring to patients' homes because of obstacles related to cultural issues or the patient's residence in nearby cities in the test group, using a ratio of the sample sizes $(r=$ 
1.3) for unequal sample sizes in groups. The sample size was 35 in the interventional group and 45 in the control group.

Initially, 98 patients were enrolled into the study, 14 patients did not have the inclusion criteria, four subjects declined to participate. The study was performed on 80 subjects allocated to the intervention and control groups by simple random allocation with using random numbers table. Two patients, one in each interventional and control group died during the study (Fig. 1)

\section{Intervention group (liaison nurse management)}

Liaison nurse management consists of 4 phases, assessment, planning, Implementation, and evaluation.

\section{Assessment phase}

Patients of both groups were visited individually on the first day of admission in the neurosurgical department to assess score of NIHSS, to have or not to have urinary catheter, nasal gastric tube (NGT), and paralysis or not as a complication of stroke. The liaison nurse assessed the patient, and the probability of pneumonia and UTI based on risk factors of each.

\section{Planning phase}

An individualized care plan was developed for each patient according to the priorities, objectives, and expected results, was determined.

\section{Implementation phase}

Patients received the nursing care according to individualized patient's needs. Care was carried out individually by the researcher for each patient. One of the family members who were more empowered to care for the patient was received the necessary training for home care. Care and instructions required based on guidelines and reliable sources, was done. Training to the patient's family on the patient's bedside is done within 2 days of admission (the first day of oral education and the second day in practical form). The patient and his/her family were given a booklet containing guidelines for patient care in patients with stroke upon discharge from the hospital.

The patient or family of the patient was given a telephone number to communicate with the liaison nurse to resolve potential problems by coordinating the nurse.

After discharge from hospital, the liaison nurse visited the patient at home two sessions per week lasting 45-60 minutes for one month and provided necessary interventions aiming at resolving their caring issues.

Nursing care primarily focused on educating the patient and his family, including the basic concepts of stroke, the definition and nature of the disease, the types, causes, warning signs, and effects of stroke on 
the whole body. The care plan for each of the complications of Pneumonia and UTI are listed in below box.

Pneumonia

- Effective cough and deep breathing.

- Getting out of the bed as soon as possible.

- Chest physiotherapy in coordination with the physiotherapist and suctioning according to the patient's need.

- Chest physiotherapy by the family or caregiver at home.

- Semi-sitting position after feeding to prevent aspiration.

- Teaching the family how to eat at home or take care of the patient.

- Oral hygiene using Chlorhexidine solution.

- Educating pneumonia symptoms including fever, decreased consciousness, and shortness of breath, increased sputum.

UTI

- Not using the Foley catheter in case of urinary incontinence and the use of diapers for adults.

- Educating the family about the hygiene of the perinea area and replacing the patient diaper at short intervals.

- Use of a temporary catheter for urinary retention at intervals.

- Teaching the patient's family for caring a permanent urinary catheter (keeping the urine bag intact and washing the area at home).

- Ensuring the adequacy of the fluid intake by encouraging the patient to take fluids and controlling the amount of venous fluid.

- Educating about UTI including fever, decreased consciousness, bad breath, and dysuria.

\section{Evaluation phase}

Both study and control groups were followed up two weeks and two months after discharge for the incidence of pneumonia and UTI. In the event of symptoms that could cause a UTI or pneumonia, before two weeks and two months, the patients referred for visit the physician; the liaison nurse provided the necessary coordination to visit the clinic and visit the neurologist.

\section{Control group (standard care)}

Patients in the control group as well were visited individually on the first day of admission to determine the NIHSS and having urinary catheter, NGT, and condition of paralysis as a complication of stroke. The control group patients were under routine hospital care. The control group was evaluated for pneumonia and UTI as in the intervention group, two weeks and two months after discharge from the hospital. 


\section{Occurrence of complications}

In this study, the occurrence of pneumonia was determined based on symptoms of fever, decreased consciousness, dyspnea, sputum, and positive result of sputum culture, and UTI based on symptoms of fever, bad smell of urine, dysuria, and decreased consciousness level. However, it was up to the treating physician to confirm the nurse's diagnosis.

\section{Data analysis}

Data analysis was conducted by an expert statistician blinded to the study protocol. The collected data were analyzed by SPSS 20 , using Chi-square and independent $T$ tests. $P<0.05$ was considered statistically significant.

\section{Results}

In this study, 80 patients were examined, 35 patients in the intervention and 45 patients in the control group with mean ages of $64 \pm 10$ and $66 \pm 08$ years respectively $(P=0.37)$. Results of two months after discharge are for 78 patients (34 in intervention and 44 in control groups), because of dying two patients (one in each group) between two times of evaluation.

Considering the randomized distribution of the patients into the intervention and control groups, two groups had no statistically significant differences in terms of sex, age groups, having nasalgastrointestinal tube, urinary catheter, and paralysis (Table 1). 
Table 1

The patients' characteristics in the intervention and control groups

\begin{tabular}{|c|c|c|c|c|}
\hline & Group & & $x^{2} / t$ & $P$ value \\
\hline & Intervention $(n=35)$ & Control $(n=45)$ & & \\
\hline & $\mathbf{N}(\%)$ & $\mathbf{N}(\%)$ & & \\
\hline Age (years): & $18(51.4)$ & $19(42.2)$ & 0.671 & $0.413^{\star}$ \\
\hline$\leq 65$ & $17(48.6)$ & $26(57.8)$ & -1.003 & $0.319 * \star$ \\
\hline$>65$ & $45-85$ & $49-83$ & & \\
\hline $\begin{array}{l}\text { Range } \\
\text { mean } \pm S D\end{array}$ & $64.89 \pm 10.19$ & $66.98 \pm 08.44$ & & \\
\hline Sex: & $19(54.3)$ & $23(51.1)$ & 0.080 & $0.778^{\star}$ \\
\hline Male & $16(45.7)$ & $22(49.8)$ & & \\
\hline Female & & & & \\
\hline NIHSS: & $12(34.3)$ & $14(31.1)$ & 0.133 & $0.936^{\star}$ \\
\hline $16-18$ & $14(40.0)$ & $1840.0)$ & -0.131 & $0.896^{\star \star}$ \\
\hline $19-22$ & $9(25.7)$ & $13(28.9)$ & & \\
\hline $23-25$ & $16-25$ & $16-25$ & & \\
\hline $\begin{array}{l}\text { Range } \\
\text { mean } \pm S D\end{array}$ & $20.40 \pm 2.96$ & $20.49 \pm 3.05$ & & \\
\hline Having NGT: & $18(51.4)$ & $20(44.4)$ & 0.385 & $0.535^{\star}$ \\
\hline Yes & $17(48.6)$ & $25(55.6)$ & & \\
\hline No & & & & \\
\hline Having Urinary Catheter: & $21(60.0)$ & $27(60.0)$ & 0.000 & $1.000 *$ \\
\hline Yes & $14(40.0)$ & $18(40.0)$ & & \\
\hline No & & & & \\
\hline
\end{tabular}

\footnotetext{
* Chi-Square test

$\star \star t-$ test
} 


\begin{tabular}{|c|c|c|c|c|}
\hline & \multicolumn{2}{|l|}{ Group } & \multirow[t]{3}{*}{$X^{2} / t$} & \multirow[t]{3}{*}{$P$ value } \\
\hline & Intervention $(n=35)$ & Control $(n=45)$ & & \\
\hline & $\mathbf{N}(\%)$ & $\mathbf{N}(\%)$ & & \\
\hline Having paralysis: & $28(80.0)$ & $35(77.8)$ & 0.062 & $0.969 *$ \\
\hline One side & $2(5.7)$ & $3(6.7)$ & & \\
\hline Two sides & $5(14.3)$ & $7(15.6)$ & & \\
\hline \multicolumn{5}{|l|}{ None } \\
\hline \multicolumn{5}{|l|}{ * Chi-Square test } \\
\hline$\star \star t$ - test & & & & \\
\hline
\end{tabular}

Two weeks and two months after discharge, in the intervention group, $5.7 \%$ and $5.9 \%$ of the patients, and in the control group $11.1 \%$ and $9.1 \%$ of the patients, suffered from pneumonia. There was no statistically significant difference between the two groups in the incidence of pneumonia two weeks $(P=0.45)$ and two months after discharge $(P=0.69)$ and in total between the groups $(11.6$ vs. $19.2, P=0.35)$.

The incidence of UTIs two weeks and two months after discharge in the intervention group was $0 \%$. Also, in the control group, it was $6.7 \%$ and $15.9 \%$, respectively. The incidence of UTI in the two groups was not statistically significant two weeks $(P=0.25)$, but this difference was significant two months after the discharge $(P=0.01)$ and in total between the groups $(P<0.001)($ Table 2$)$.

Table 2

Incidence of complications in the two groups after discharging from hospital.

\begin{tabular}{|lllll|}
\hline Complication & & $\begin{array}{l}\text { Intervention } \\
(\mathbf{n}=\mathbf{3 5}, \mathbf{3 4})\end{array}$ & $\begin{array}{l}\text { Control } \\
(\mathbf{n}=\mathbf{4 5}, \mathbf{4 4})\end{array}$ & P value* \\
& & $\mathbf{N}(\%)$ & $\mathbf{N}(\%)$ & \\
\hline Pneumonia & After 2 weeks & $2(5.7)$ & $5(11.1)$ & 0.39 \\
& After 2 months & $2(5.9)$ & $4(9.1)$ & 0.59 \\
& Total & $4(11.6)$ & $9(19.2)$ & 0.35 \\
\hline UTI & After 2 weeks & $0(0)$ & $3(6.7)$ & 0.11 \\
& After 2 months & $0(0)$ & $7(15.9)$ & 0.01 \\
& Total & $0(0)$ & $10(24.6)$ & $<0.001$ \\
\hline *Chi-square test & & & \\
\hline
\end{tabular}




\section{Discussion}

To prevent post-stroke complications, the function of the liaison nurse can help reduce their problems. Stroke patients and their family face many problems after discharge, do not have normal life and are dependent on others. Nurses are in an ideal position to help in these situations, because they are constantly in clinical practice and are a mediator between the patient, family, and health care team (22).The aims of liaison nursing are to improve the communications between hospital and home care and continuity and consequently improve the quality of care (12).

In this study, the effect of the liaison nurse performance on the prevention of the occurrence of infections stroke complications including pneumonia and UTI, was investigated.

Infections complications, most often pneumonia, contribute to increased mortality from stroke, prolong hospitalization, difficulties in care, reduce functional performance improvement and increased the cost of treatment $(23$.

The results of this study showed that the rate of pneumonia in liaison nurse group in two months was $11.6 \%$ less than in control group (19.2\%). The incidence of complications from stroke is different in studies. It seems that the length of follow-up periods is effective in these differences. In a six-year period study of in-hospital stroke complications, reported the incidence of pneumonia to be $9 \%$ (8). A multicentre study confirmed that at seven days after the acute stroke onset pneumonia occurred in $7.4 \%$ patients while during the first three months occurred in $13.6 \%$ of patients (24). Other studies presented a range of incidence for pneumonia regardless to follow up period: $10-20 \%(25), 1-33 \%(23)$, and $7-22 \%(26)$.

To prevent of aspiration pneumonia, the liaison nurse with practical education on how to feed the patient with a gastric tube, usually on the last day of hospitalization and during discharge, sought to increase the awareness of the family care givers. Care givers must be assessed for their capacity to provide the needed care and their readiness to assume the care giving role at home (2). We found that Involving the caregiver from the first day of admission prevent confusion and can provide better living conditions for the patient and his/her family. The results of this study showed that the presence of the liaison nurse in the patient's bedside, education of the correct way and engaging the patient's caregiver for suction, oral hygiene, mouthwash and tooth brushing that usually less considered after discharge, can reduce the incidence of pneumonia.

UTI, another commonly reported complication after stroke, has been shown to be associated with a poor outcome and mortality in the patient with stroke (27).

The results of this study showed that liaison nursing care resulted in a significant difference in the incidence of UTI between the groups.

The majority of UTIs in acute stroke are associated with the use of indwelling catheters; therefore prolonged ones should be avoided. The risk of UTI is $3-10 \%$ per day of catheterization, approaching $100 \%$ after 30 days. Urinary incontinence and retention are common after stroke (29-58\%), and limited 
mobility increases the likelihood of being catheterized (7). The use of a Foley catheter for more than 48 hours after stroke increases the risk of urinary tract infection (20).

Despite the efforts of the liaison nurse for preventing our patients from having a permanent urinary catheter with a low level of consciousness, obtaining the consent of the physician for inserting a temporary catheter, and teaching how to insert it to the patient's family, a limited number of family caregivers were willing to do the procedure. Although programs are designed to train family members in caring skills, they often lack the preparation and support to take on a caring role (2).

In this study, we obtained the better results in relation to the performance of liaison nurse in prevention of UTI. Although most patients had a permanent catheter, the incidence of UTI is $0 \%$ over the entire two month follow up period in the intervention group. In other studies, the incidence of UTI was reported to be $11 \%$ (27), $2-27 \%(23), 16-27 \%(7)$, and $7-28 \%$ (26), or even up to $43 \%$.

Most studies have reported incidence of post-stroke complications, and few interventions have been conducted to prevent these complications. Some studies also examine the impact of community-based nursing interventions on other aspects of stroke patients' lives. A randomized controlled trial of a nurseled community-based self management program for improving recovery among community-residing stroke survivors reported that 4 weeks program including home visit and follow-up phone calls improving self-efficacy, outcome expectation, and performance of stroke self-management behaviors (29). In another study, tested phone-based intervention under nurse guidance after stroke is effective in improving blood pressure control and medication adherence among Ghanaian stroke patients within 1 month of symptom onset compared with standard of care (30).

In our study, once again, shown involving the family in the care of stroke patients has good results. Recommend that the family/caregiver of the stroke patient, as essential members of the rehabilitation team, should be informed and involved in decision making and treatment planning as early as possible, and throughout the duration of the rehabilitation process (20).

To the best of the authors' knowledge, this is the first randomized controlled trial which examines the liaison nurse performance among Iranian community-residing stroke survivors.

Since this project was a student thesis, we were faced with a time and budget limit. It seems that in order to determine the effect of interface liaison nurse performance in stroke patients, designing and conducting long-term and large-scale studies may produce different results and more persuasive evidence. The other limitations of this study were convenience sampling and also because of the nature of the study, it was not possible for patients to be blinded to being in the intervention or control group.

Education and most importantly, not leaving the patient alone after the discharge, continuing care and monitoring of the patient at home are interventions by the liaison nurse.

\section{Conclusion}


The results of this study showed that with liaison nurse performance, there was a significant difference in the incidence of UTI, in stroke patients in two months after discharge from hospital, but the incidence of pneumonia had no statistically significant differences in two groups. This study showed the nurse's evaluation each patient individually according to her/his needs, developing and monitoring the homebased care program, beyond overall education to these patients, could reduce the complications of a stroke.

\section{Abbreviations}

UTI

Urinary Tract Infection; NIHSS:National Institutes of Health Stroke Scale; NGT:Nasal Gastric Tube.

\section{Declarations}

Ethics approval and consent to participate: This study was designed due to the Declaration of Helsinki and compliance with the principle of confidentiality and anonymity, approved by the ethics committee of Shahid Sadoughi University of Medical Sciences, Iran (code number: IR.SSU.REC.1396.43). The necessary explanations were given to the patients and their families about the study process, its goals and conditions. Participation in the study was entirely voluntary. Written informed consent was signed by eligible patients who were willing to participate in the study.

Consent for publication: Not applicable.

Availability of data and materials: The datasets used and/or analysed during the current study are available from the corresponding author on reasonable request.

Competing interests: The authors declare that they have no competing interest.

Funding: This article has been extracted from master's thesis of Sedigheh Ebrahimi in Intensive Nursing Care, was supported financially by Shahid Sadoughi University of Medical Sciences, Yazd, Iran.

Authors' contributions: Study design: ZK and SE; data collection: SE; data analysis: ZK, SE, HF; drafting of manuscript: ZK, SE, HF; statistical analysis: HF; supervision: ZK. All authors read and approved the final manuscript.

Acknowledgements: The authors gratefully acknowledge the patients and their families who took part in this study.

\section{References}

1. - Fahimfar N, Khalili D, Mohebi R, Azizi F, Hadaegh F. Risk factors for ischemic stroke; results from 9 years of follow-up in a population based cohort of Iran. BMC neurology. 2012;12(1):1-7. 
2. -Camicia M, Lutz BJ. Nursing's role in successful transitions across settings. Stroke. 2016;47(11):e246-9. https://doi.org/10.1161/STROKEAHA.116.012095

3. - Azarpazhooh MR, Etemadi MM, Donnan GA, Mokhber N, Majdi MR, Ghayour-Mobarhan M, et al. Excessive incidence of stroke in Iran: evidence from the Mashhad Stroke Incidence Study (MSIS), a population-based study of stroke in the Middle East. Stroke. 2010;41(1):e3-10.

4. - Sacco RL, Kasner SE, Broderick JP, Caplan LR, Connors JJ, Culebras A, et al. American Heart Association Stroke Council, Council on Cardiovascular Surgery and Anesthesia; Council on Cardiovascular Radiology and Intervention; Council on Cardiovascular and Stroke Nursing; Council on Epidemiology and Prevention; Council on Peripheral Vascular Disease; Council on Nutrition, Physical Activity and Metabolism. An updated definition of stroke for the 21st century: a statement for healthcare professionals from the American Heart Association/American Stroke Association. Stroke. 2013;44(7):2064-89. https://doi.org/10.1161/STR.0b013e318296aeca.

5. - Armstrong M. Postdischarge nursing care of stroke patients. American nurse today. 2014; https://www.americannursetoday.com/postdischarge-nursing-care-of-stroke-patients. Accessed July 2020.

6. - Condon C, Lycan S, Duncan P, Bushnell C. Reducing readmissions after stroke with a structured nurse practitioner/registered nurse transitional stroke program. Stroke. 2016;47(6):1599-604. https://doi.org/10.1161/STROKEAHA.115.012524

7. - Indredavik B, Rohweder G, Naalsund E, Lydersen S. Medical complications in a comprehensive stroke unit and an early supported discharge service. Stroke. 2008;39(2):414-20. https://doi.org/10.1161/STROKEAHA.107.489294

8. - Ingeman A, Andersen G, Hundborg HH, Svendsen ML, Johnsen SP. In-hospital medical complications, length of stay, and mortality among stroke unit patients. Stroke. 2011;42(11):3214-8. https://doi.org/10.1161/STROKEAHA.110.610881.

9. - Almeida SR, Bahia MM, Lima FO, Paschoal IA, Cardoso TA, Li LM. Predictors of pneumonia in acute stroke in patients in an emergency unit. Arquivos de neuro-psiquiatria. 2015;73(5):415-9. https://doi.org/10.1590/0004-282X20150046

10. - Poisson SN, Johnston SC, Josephson SA. Urinary tract infections complicating stroke: mechanisms, consequences, and possible solutions. Stroke. 2010 Apr 1;41(4):e180-4. https://doi.org/10.1161/STROKEAHA.109.576413.

11. - Rennke S, Ranji SR. Transitional care strategies from hospital to home: a review for the neurohospitalist. The Neurohospitalist. 2015;5(1):35-42. https://doi.org/10.1177/1941874414540683

12. - Arts SE, Francke AL, Hutten JB. Liaison nursing for stroke patients: results of a Dutch evaluation study. Journal of Advanced Nursing. 2000;32(2):292-300.

13. - Burton C, Gibbon B. Expanding the role of the stroke nurse: a pragmatic clinical trial. Journal of Advanced Nursing. 2005;52(6):640-50. https://doi.org/10.1111/j.1365-2648.2005.03639.x 
14. - Nasrabad RR. Introducing a new nursing care model for patients with chronic conditions. Electronic physician. 2017;9(2):3794. https://doi.org/dx.doi.org/10.19082/3794

15. - Ribas EDN, Bernardino E, Larocca LM, Poli Neto P, Aued GK, Silva CPCD. Nurse liaison: a strategy for counter-referral. Revista brasileira de enfermagem. 2018;71, 546 - 53. https://doi.org/10.1590/00347167-2017-0490

16. - Tabanejad Z, Pazokian M, Ebadi A. The effect of liaison nurse service on patient outcomes after discharging from ICU: A randomized controlled trial. Journal of caring sciences. 2016;5(3):215. https://doi.org/10.15171/jcs.2016.023

17. - Van Emden DM, Ros WJ, Berns MP. Transition of care: an evaluation of the role of the discharge liaison nurse in the Netherlands. Journal of Advanced Nursing. 1999;30(5):1186-94. https://doi.org/10.1046/j.1365-2648.1999.01190.x

18. - Alberto L, Zotárez H, Cañete ÁA, Niklas JE, Enriquez JM, Gerónimo MR, del Carmen Martínez M, Chaboyer W. A description of the ICU liaison nurse role in Argentina. Intensive and Critical Care Nursing. 2014;30(1):31-7. https://doi.org/10.1016/j.iccn.2013.07.001.

19. - Tabanejad Z, Pazokian M, Ebadi A. A systematic review of the liaison nurse role on patient's outcomes after intensive care unit discharge. International journal of community based nursing and midwifery. 2014;2(4):202.

20. - Duncan PW, Zorowitz R, Bates B, Choi JY, Glasberg JJ, Graham GD, Katz RC, Lamberty K, Reker D. Management of adult stroke rehabilitation care: a clinical practice guideline. stroke. 2005;36(9):e10043. https://doi.org/10.1161/01.STR.0000180861.54180.FF

21. - Kazemnejad-Leili E, Rezaei S, Hosseini-Nejad M, Bakhshayesh-Eghbali B, Saberi A, Keshavarz P. The applicability, concurrent validity and internal consistency reliability of the Persian version of the National Institutes of Health Stroke Scale (NIHSS): evidences for gender differences. Caspian Journal of Neurological Sciences. 2016;2(1):18-28.

22. - Kalani Z, Pourkermanian P, Alimohammadi N. The effect of family guided visits on the level of consciousness in traumatic brain injury. Journal of Biology and Today's World. 2016; 5(5), 86-90. https://doi.org/10.15412/J.JBTW. 01050502

23. - Małodobry K, Juda M, Ozga D. Pneumonia as an impediment to treat patients in the early period after stroke. Journal of Education, Health and Sport. 2018;8(8):723-34. doi: org/10.6084/m9.figshare.6983207

24. - Aslanyan S, Weir CJ, Diener HC, Kaste M, Lees KR, GAIN International Steering Committee and Investigators. Pneumonia and urinary tract infection after acute ischaemic stroke: a tertiary analysis of the GAIN International trial. European journal of neurology. 2004;11(1):49-53.

25. - Chen LF, Chang CY, Hsu LC, Tsai PH, Chang SJ, Chang SC, Yuan MK, Lai YC, Liu YC, Wang WS. Bacterial pneumonia following acute ischemic stroke. Journal of the Chinese Medical Association. 2013;76(2):78-82. https://doi.org/10.1016/j.jcma.2012.10.005

26. - Lee J. Management of acute stroke complication. Journal of the Korean Medical Association. 2009;52(4):365-74. https://doi.org/10.5124/jkma.2009.52.4.365

Page $14 / 16$ 
27. - Bogason E, Morrison K, Zalatimo O, Ermak DM, Lehman E, Markley E, Cockroft K. Urinary tract infections in hospitalized ischemic stroke patients: source and impact on outcome. Cureus. 2017;9(2). https://doi.org/10.7759/cureus.1014

28. - Teasell R, Foley N, Salter K, Bhogal S, Jutai J, Speechley M. Evidence-based review of stroke rehabilitation: executive summary. Topics in stroke rehabilitation. 2009;16(6):463-88. https://doi.org/10.1310/tsr1606-463.

29. - Lo SH, Chang AM, Chau JP. Study protocol: a randomised controlled trial of a nurse-led communitybased self-management programme for improving recovery among community-residing stroke survivors. BMC Health Serv Res. 2016;16(1):387. https://doi.org/10.1186/s12913-016-1642-9

30. - Sarfo FS, Treiber F, Jenkins C, Patel S, Gebregziabher M, Singh A, Sarfo-Kantanka O, Saulson R, Appiah L, Oparebea E, Ovbiagele B. Phone-based Intervention under Nurse Guidance after Stroke (PINGS): study protocol for a randomized controlled trial. Trials. 2016;17(1):436. https://doi.org/10.1186/s13063-016-1557-0

\section{Figures}




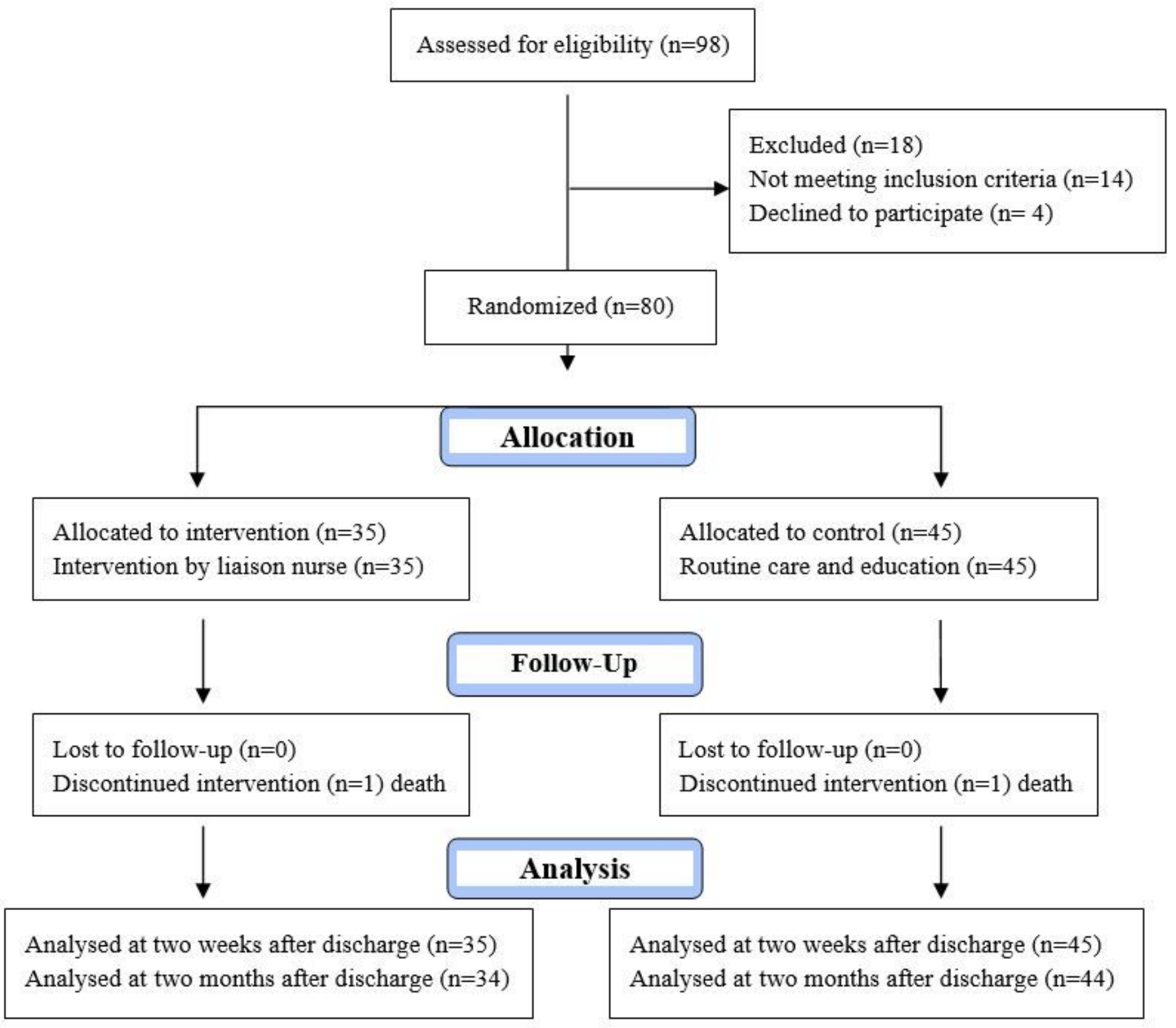

\section{Figure 1}

Flowchart of the study 